JOURNAL

of Health Inequalities

\title{
The future of treatment of tobacco dependence - harnessing technology to deliver hybrid interventions
}

\author{
Aleksandra Herbeć \\ Health Promotion Foundation, Nadarzyn, Poland \\ Centre for Behaviour Change, Clinical Educational, and Health Psychology, UCL, London, UK
}

Supplementary materials (slides) are available in Webappendix 16 at the journal's website: https://www.termedia.pl/Journal/Journal_of_ Health_Inequalities-100.

Over the past decades many high-income countries have witnessed marked declines in the prevalence of smoking among adults and children. This success could be attributed to several factors, including evidence-based tobacco control programmes, as well as supranational initiatives and grassroots movements, energised by the World Health Organization (WHO) Framework Convention on Tobacco Control (FCTC). Nonetheless, 1 billion people globally continue to smoke. While the majority of smokers want to quit and make frequent attempts to do so, stronger strategies are needed to increase abstinence rates.

There has been a growing offer in terms of on-prescription and over-the-counter pharmacotherapy supporting smoking cessation. More recently we have seen the emergence of next-generation diverse nicotine-delivery devices that might aid cessation, at least among some smokers. In countries like Poland we have also observed a rekindled interest among smokers in pharmacological support, which could at least partially be attributed to vigorous marketing and promotional campaigns, especially on television. Indeed, around one million packages of cessation medications were sold in Poland in 2014 and 2015, primary of cytisine-based drugs.

However, there are important limitations to the many cessation programmes. First of all, access to affordable and evidence-based cessation support is very limited in many countries, and only a small minority of smokers in Europe accesssuchsupport.Secondly, adherencetobothbehavioural as well as pharmacological support is low, which is likely to hinder success.

Digital interventions have long held promise to offset some of the limitations of traditional cessation support, including to extend the reach of cessation programmes. We have evidence that many forms of self-help interventions can aid cessation, including printed materials, mobilebased support (e.g. SMS texting), as well as computer- and Internet-based support. More recently, interest has shifted towards smartphone-based programmes for quitting smoking that offer ever more diverse opportunities to intervene, collect data, and personalise the support offered. However, evidence on their effectiveness remains scarce, which is also due to the challenges of conducting well controlled studies in this field. Moreover, technologically-supported cessation programmes face two key challenges: low uptake among the general population, and high attrition and low engagement that may be insufficient to generate an effect.

Successful harnessing of technology to support smoking cessation is likely to require approaches that address the barrierstouseandadherencetosuch programmes. Theywill also need to be accompanied by effective implementation strategies. This is especially important within healthcare systems, which engage and support vulnerable and priority populations of smokers that could benefit most from cessation in the immediate future.

Healthcare professionals can play a pivotal role in the delivery of evidence-based and technology-assisted cessation support to their patients and their families. A new programme, STAR (Smoking Treatment Advisory Resource), which will soon be available in Poland, has been developed to support hybrid interventions that can combine expert input by clinicians with digitallydelivered assistance. Research on such programmes is required to help determine how to best implement and develop them further.

Key words: cigarette smoking, digital health, healthcare system.

\section{DISCLOSURE}

The author has received unrestricted funds as part of Global Bridges at Mayo Clinic and Pfizer Independent Grants for Learning and Change Request for Proposals: EUROPEAN PROGRAM to develop and evaluate programme STAR, and researches the use of technology in smoking cessation. 